\title{
Evaluation of Surface Properties of Four Tooth-Colored Restorative Materials
}

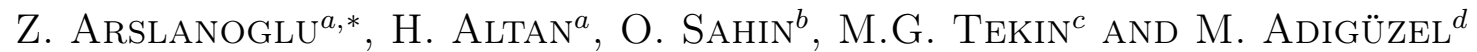 \\ ${ }^{a}$ Mustafa Kemal University, Faculty of Dentistry, Pediatric Dentistry Department Hatay, Turkey \\ ${ }^{b}$ Mustafa Kemal University, Science and Art Faculty, \\ Micro/Nanomechanic Characterization Laboratuary, Hatay, Turkey \\ ${ }^{c}$ Mustafa Kemal University, Faculty of Dentistry, Conservative Treatment Department Hatay, Turkey \\ ${ }^{d}$ Mustafa Kemal University, Faculty of Dentistry, Endodontic Department Hatay, Turkey
}

\begin{abstract}
The aim of this study is to compare microhardness, roughness and micromorphology of the examined materials of a newly developed glass carbomer dental filling material to two different resin modified glass ionomers and a glass ionomer dental filling material. Specimens tested were prepared from a glass carbomer without gloss (Glass Carbomer, GCP), a glass carbomer (Glass Carbomer, GCP) with gloss, two different resin modified glass ionomers (Fuji II LC, GC and Riva LC, SDI) and a glass ionomer (Equia, GC). Seven specimens of each material were prepared according to manufacturer's recommendations. After setting, samples were polished and stored in $37^{\circ} \mathrm{C}$ distilled water for $24 \mathrm{~h}$. Indentation microhardness of examined restorative materials was measured using the Vickers indenters. In addition surface roughness of the materials was measured using surface profilemeter. Surface morphology was analyzed by using scanning electron microscopy. There was a significant difference in microhardness and roughness between the restorative materials $(p<0.05)$. The highest microhardness was measured for Equia and lowest for glass carbomer with gloss. The roughness was fully definite for the glass carbomer with gloss and indefinite for the glass carbomer without gloss. Small cracks and voids were observed in all groups. High viscosity glass ionomer cement Equia exhibits the high physical strength than RMGIC. Gloss application does not influence the hardness behavior of GCP.
\end{abstract}

DOI: 10.12693/APhysPolA.128.B-310

PACS: 87.85.J-, 62.20.-x

\section{Introduction}

Glass ionomer cements (GIC) were introduced to dentistry in the early 1970's, and have been widely used in restorative dentistry because of advantages such as biocompatibility, adhesion to dental hard tissues, and fluoride release $[1,2]$. However, these materials have some clinical limitations, including a prolonged setting time, moisture sensitivity during initial setting, dehydration, and rough surface character, which can block mechanical resistance $[1,3]$.

In order to overcome these drawbacks, resin-modified glass ionomer cements (RMGICs) were developed. In addition to the GIC formulation, these materials contain monomers and photo-initiators. These RMGICs set faster and have a higher viscosity because of the presence of finer glass particles, anhydrous polyacrylic acids of high molecular weight, and a high powder-to-liquid mixing ratio. The setting reaction is the same as the acid-base reaction of a typical, conventional GIC.

Recently, glass carbomer cement, a GIC based restorative material, has been introduced with claims of improved physical characteristics [4]. This new material contains nanosized glass particles and fluorapatite as

*corresponding author; e-mail: halenuronat@gmail.com a filler. The reactive glass is activated with dialkyl siloxane as described in the European Patent, number 20040748628 [5].

The aim of this study is to compare the microhardness, roughness, and micromorphology of the examined materials of a newly developed glass carbomer dental filling material to two different resin modified glass ionomers, and a glass ionomer dental filling material.

\section{Materials and methods}

\subsection{Vickers hardness}

The current study tested 4 different dental filling materials in 5 Groups with 4 specimens of each. Specimens tested were prepared from a glass carbomer without gloss (Glass Carbomer, GCP), glass carbomer (Glass Carbomer, GCP) with gloss, two different resin modified glass ionomers (Fuji II LC, GC and Riva LC, SDI) and a glass ionomer (Equia, GC).

Twenty $10 \mathrm{~mm}$-thick parallel PMMA cylinders with $50 \mathrm{~mm}$ diameter were fabricated. In the center of one of the flat surfaces of each cylinder block a $2 \mathrm{~mm}$-deep and $10 \mathrm{~mm}$-wide circle cavity was prepared and cyanoacrylate $(\mathrm{CA})$ adhesive treatment was applied on all of its margins.

PMMA blocks were randomly divided into 5 groups of 4 cylinders each, and any cavity was filled with one of the tested dental filling materials, according to their group. 
Group 1. Riva LC (SDI, Australia) was used to fill the cavities; a capsule mixer (Linea Tac 400M) was used for $10 \mathrm{~s}$ of mixing prior to the application of the material with a Riva Applicator. The specimens were subjected to a $1400 \mathrm{~mW}$ light-heating procedure (GCP Carboled Lamp) for $20 \mathrm{~s}$ of light-curing.

Group 2. Equia (GC, Japan) was used to fill the cavities; a capsule mixer (Linea Tac 400M) was used for $10 \mathrm{~s}$ of mixing prior to the application of the material with a Fuji Applicator. The specimens were cover-layered with a Fuji Coat and were subjected to a $1400 \mathrm{~mW}$ lightheating procedure (GCP Carboled Lamp) for $20 \mathrm{~s}$ during the chemical setting process.

Group 3. Fuji II LC (GC, Japan) was used to fill the cavities; a capsule mixer (Linea Tac 400M) was used for $10 \mathrm{~s}$ of mixing prior to the application of the material with a Fuji Applicator. The specimens were subjected to a $1400 \mathrm{~mW}$ light-heating procedure (GCP Carboled Lamp) for $20 \mathrm{~s}$ of light-curing.

Group 4. Glass Carbomer (GCP, The Netherlands) was used to fill the cavities; a capsule mixer (Linea Tac $400 \mathrm{M}$ ) was used for $10 \mathrm{~s}$ of mixing prior to the application of the material with a GCP carboCAP Applicator. The specimens were subjected to a $1400 \mathrm{~mW}$ light-heating procedure (GCP Carboled Lamp) for $60 \mathrm{~s}$ of light-curing.

Group 5. Glass Carbomer (GCP, Netherland) was used to fill the cavities; a capsule mixer (Linea Tac 400M) was used for $10 \mathrm{~s}$ of mixing prior to the application of the material with a GCP carboCAP Applicator. The specimens were cover-layered with GCP gloss and were subjected to a $1400 \mathrm{~mW}$ light-heating procedure (GCP Carboled Lamp) for $60 \mathrm{~s}$ of light-curing.

Twenty-three hours after the application of each specimen, irregularities on the surfaces due to manual application procedures were smoothed mechanically by grinding with 1200 and 2400 grit $(\mathrm{SiC})$ sandpaper, and then polished on 6,3 , and $1 \mu \mathrm{m}$ diamond lap wheels, respectively. Each specimen was subjected to the Vickers test 3 times, just after 24 hours of material application. The Vickers indenter was used with a load of $9.8 \mathrm{~N}$, and the loading time was $15 \mathrm{~s}$ at room temperature.

The Vickers microindentation hardness values (i.e., $H_{V}$ ) were calculated using the standard formula:

$$
H_{v}=1.8544 \frac{P}{d^{2}}
$$

where $P$ is the applied test load in N, $d$ is the average of two indentation diagonal lengths in $\mu \mathrm{m}$, and 1.8544 is the geometrical constant of the diamond pyramid.

\subsection{Surface roughness}

The surface roughness of the filling materials was measured using a mechanic roughness measurement device (Taylor Hobson-Surtronic 25, UK). Each specimen was tested for surface finished at 3 different point regions.

\subsection{SEM analysis}

Cylindrical specimens $10 \mathrm{~mm}$ in diameter and $2 \mathrm{~mm}$ in height were prepared. One sample was selected from each group. The samples were viewed without gold coating under the SEM. Micrographs at x35 magnification were obtained to assess the surface change of the materials.

\subsection{Statistical analysis}

For Vickers Hardness test one-way analysis of variance (ANOVA) was used to statistically analyze data at the $p<0.05$ level of significance. The distirubations of samples were analyzed with Kolmogrov-Smirnov $Z$ test. For roughness test Mann Whitney $U$ test was used to statistically analyze the data at the $p<0.05$ level of significance analysis was conducted with the SPSS statistical package (Statistical Package for the Social Science; SPSS Inc, Chicago, IL, USA).

\section{Results and discussion}

The mean value of the GCP without the gloss was measured as $61.262 \mathrm{MPa}$. Although that value was greater than the mean value of the GCP with gloss, no statistical difference was found between the results $(p>0.05)$. The mean and standard deviations of the surface hardness values are shown in Table. The hardness values of the tested materials for all of the groups were found to be statistically significant $(p<0.05)$.

The mean and standard deviations of the surface roughness $(\mathrm{Ra})$ are shown in Table. There were statistically significant differences between the groups $(p<$ $0.05)$, while the smoothest surface was observed in the GCP without gloss. The Fuji II LC and GCP with gloss had the highest roughness values.

TABLE I

Mean values and standard deviations (SD) of Vickers hardness and roughness.

\begin{tabular}{l|c|c}
\hline \hline \multicolumn{1}{c|}{ Group: } & $\begin{array}{c}\text { Vickers hardness } \\
\text { mean+SD }\end{array}$ & $\begin{array}{c}\text { Roughness } \\
\text { mean }+\mathrm{SD}\end{array}$ \\
\hline 1: (Riva LC) & $583.44 \pm 63.85^{b}$ & $1.06 \pm 0.2^{a b}$ \\
2: (Equia) & $870.77 \pm 18.53^{a}$ & $1.10 \pm 0.46^{a b}$ \\
3: (Fuji II LC) & $739.38 \pm 12.80^{a b}$ & $1.54 \pm 0.33^{b}$ \\
4: (GCP without gloss) & $612.62 \pm 75.86^{b}$ & $0.81 \pm 0.46^{b}$ \\
5: (GCP with gloss) & $566.80 \pm 61.12^{b}$ & $1.54 \pm 0.25^{a}$ \\
\hline
\end{tabular}

${ }^{a, b}$ different superscripts in the same row indicates significant difference between groups.

The SEM photomicrographs obtained in this study presented that all restorative materials presented voids and cracks on their surface Fig. 1.

Laboratory studies allow the testing of anticipated variables for a better understanding of material behavior. Even when presenting some limitations, when compared to clinical conditions, in vitro studies are necessary to provide the beneficial data of new dental materials. 
For example, glass carbomer cement was developed as an alternative to GIC $[4,6]$.

Surface hardness tests appear to be appropriate for evaluating that behavior against occlusal trauma. With regard to the mechanical property of hardness, Equia presented significantly higher microhardness values when compared with the GCP and Riva. This outcome can be attributed to the high powder/liquid rate and particle di- mensions. No significant difference in the Vickers hardness was observed between the Fuji II LC, Riva LC, and GCP without gloss. This result may be due to having common ingredients (e.g., glass) in the tested restorative materials. In this study, the surface coating of the GCP using the GCP gloss was not found to be effective in increasing the Vickers Hardness of the materials.

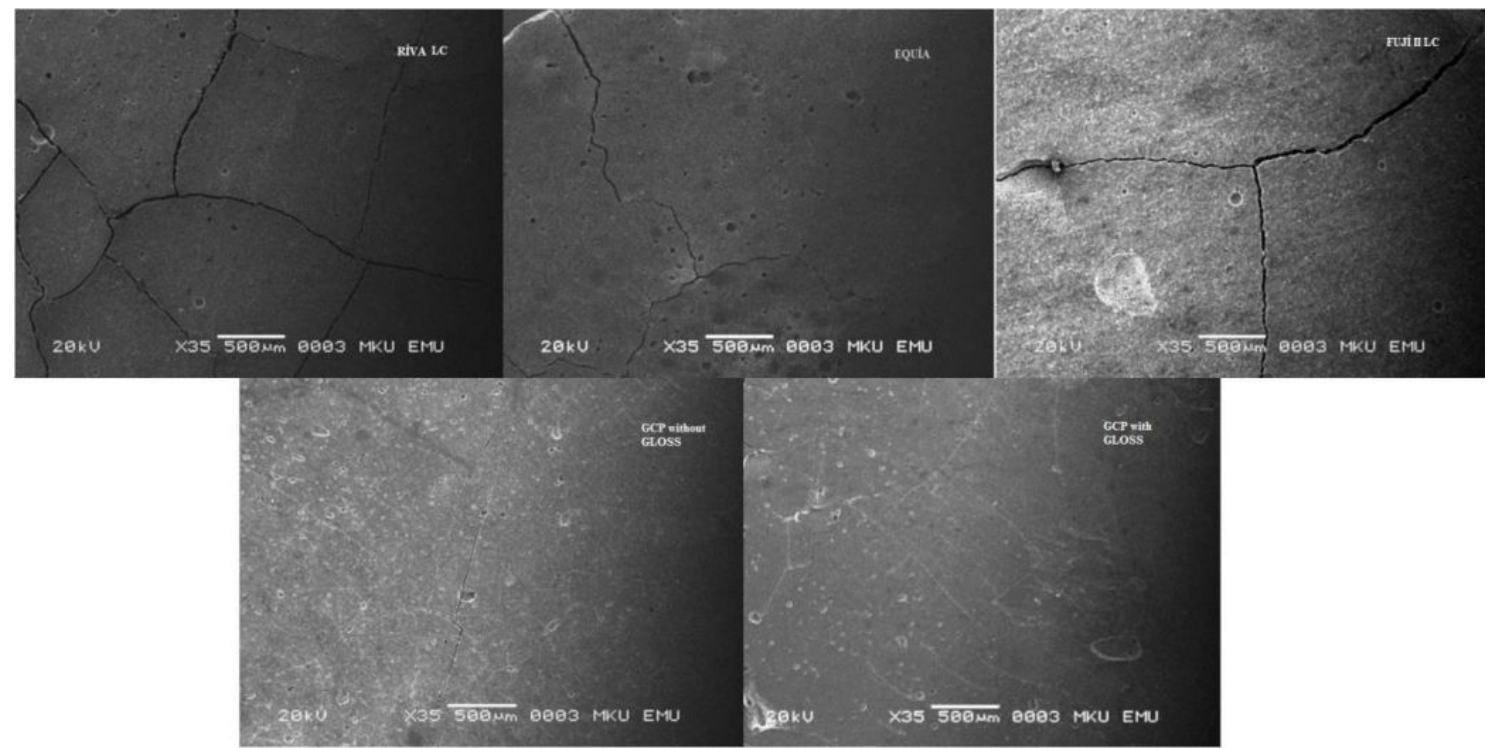

Fig. 1. Micrographs of Riva, Equia, Fuji II LC, GCP without gloss, GCP with gloss, respectively (primary magnification $\times 35)$.

One of the important objectives in finishing restoration is to achieve the smoothest surface. The surface structure is shown with a roughness value, which is the arithmetic mean of departures of the roughness profile from the mean line [7]. In this study, a polishing machine was used to adjust and standardize the pressure applied to the tested surface. In comformity with the Bollen roughness of tooth surfaces, it should be $0.20 \mu \mathrm{m}$ or lower [8]. Surface roughnesses higher than $0.20 \mu \mathrm{m}$ are likely to increase dental biofilm maturation, acidity, and bacterial adhesion, which act on material surfaces, thus increasing the risk for caries [9]. In this study, all of the materials presented surface roughnesses below this value. It was found that the GCP had less mean surface roughness when compared to the RMGIC and GIC. This may be attributed to the particle size of the materials, and the manufacturer claimed that the glass carbomer cement has nanoparticle glass enriched with fluor/hydroxyapatite.

A visual inspection of the prepared GCP samples using the gloss revealed very superficial cracks when compared to the other groups. Several close cracks were observed in the Riva group, which were caused by dehydration [10]. However, this aspect was not significantly reflected in the measured mechanical properties.

\section{Conclusion}

Therefore within the limitations of this study it can be concluded that high viscosity glass ionomer cement Equia exhibits the high physical strength than RMGIC. Gloss application does not influence the hardness behavior of GCP so without gloss application can be used to reduce the chair time. Surface behaviour of a restoration is very important to prevent the retention of microorganism form dental plaque. Glass carbomer cement has superior surface behaviours.

\section{Acknowledgments}

The financial support from research foundation of Mustafa Kemal University (grant no. 12364) is also gratefully acknowledged.

\section{References}

[1] A. Wiegand, W. Buchalla, T. Attin, Dent. Mater. 23, 343 (2007)

[2] A. Joseph, S. Abraham, S. Bhattacharji, J. Muliyil, K.R. John, N. Ethirajan, K. George, K.S. Joseph World Health Forum 9, 336 (1988). 
[3] E.A. Glasspoole, R.L. Erickson, C.L. Davidson, Dent. Mater. 18, 454 (2002).

[4] S.B. Cehreli, R.E. Tirali, Z. Yalcinkaya, Z.C. Cehreli, Eur. J. Dent. 7, 15 (2013).

[5] N.K. Thanjal, R.W. Billington, S. Shahid, J. Luo, R.G. Hill, G.J. Pearson, J. Mater. Sci-Mater. M. 21, 589 (2010).

[6] N. Zainuddin, N. Karpukhina, R.V. Law, R.G. Hill, Dent. Mater. 28, 1051 (2012).

[7] N.K. Bajwa, A. Pathak, ISRN Dent. 2014, 353926 (2014).
[8] M. Quirynen, H.C. van der Mei, C.M. Bollen, A. Schotte, M. Marechal, G.I. Doornbusch, I. Naert, H.J. Busscher, D. Van Steenberghe, J. Dent. Res. 72, 1304 (1993).

[9] O. Bala, H.D. Arisu, I. Yikilgan, S. Arslan, A. Gullu, Eur. J. Dent. 6, 79 (2012).

[10] U. Menne-Happ, N. Ilie, J. Dent. 41, 223 (2013). 